\title{
OC-043 TNF, IFNG AND FOXP3 EXPRESSION IN COLORECTAL CANCER: POTENTIAL TARGETS FOR IMMUNOTHERAPY
}

doi:10.1136/gut.2011.239301.43

S Ganapathi, ${ }^{*}$ A Beggs, S Hodgson, D Kumar St. George's University of London, London, UK

Introduction Colorectal cancer (CRC) progression is associated with suppression of host cell mediated immunity (CMI) and local immune escape mechanisms. A systemic suppression of CMI-associated cytokines, Tumour Necrosis Factor (TNF) and Interferon- $\gamma($ IFN- $\gamma$ ) has been demonstrated in patients with CRC. ${ }^{1}$ FOXP3, a crucial regulatory protein for the development and function of regulatory $\mathrm{T}$-cells could be an important cause of local immune escape mechanism in tumors. ${ }^{2}$ The aim of the study was to assess immune function in terms of the expression of TNF, IFN- $\gamma$ and FOXP3 and to identify potential targets for immunotherapy in CRC.

Methods A total of 60 colorectal cancer (CRC) patients and 15 matched controls were recruited. Total RNA was isolated from the peripheral blood mononuclear cells (PBMC, separated from a venous blood sample), fresh frozen tumour tissue and normal mucosa. TaqMan quantitative PCR (qRT-PCR) was used to determine the relative fold change in the expression of TNF, IFNG, FOXP3 in the PBMC (compared to controls) and tumour (compared to normal mucosa), with GAPDH as internal control.

Results TNF expression was suppressed in the tumour tissue (median fold change 0.48) compared to normal mucosa. IFN- $\gamma$ was found to be suppressed in the PBMC (median 0.34) compared to healthy controls. There was a significantly reduced expression of TNF ( $p=0.048)$ and IFNG $(p=0.011)$ in Stage 4 CRC. There was a significant suppression of IFNG in the recurrent CRC ( $p=0.007)$, left CRC sided CRC ( $p=0.027)$ and after neoadjuvant therapy $(p=0.021)$. The tumour tissue showed a higher expression of FOXP3 gene compared to normal mucosa (median 2.2). The expression of FOXP3 in tumour was significantly higher when the size of the tumour was more than 50 $\mathrm{mm}(\mathrm{p}=0.005)$. Late stage CRC (Stages 3 and 4) showed a significantly high expression of FOXP3 compared to early stages of CRC (Stages 1 and 2) ( $p=0.035$ ).

Conclusion The expression of TNF is suppressed and FOXP3 is enhanced in colorectal tumours and becomes more significant with tumour progression. Suppression of IFN- $\gamma$ appears to be systemic and is significantly suppressed in left sided CRC and recurrent CRC. Our study has identified potential targets which may increase the chances of response to immunotherapy based on cytokines and T regulatory cell manipulation.

Competing interests None.

Keywords FOXP3, IFNG, immunotherapy, TNF. 


\section{REFERENCES}

1. Heriot AG, Marriott JB, Cookson S, Kumar D, Dalgleish AG. Reduction in cytokine production in colorectal cancer patients: association with stage and reversal by resection. Br J Cancer 2000;82(5):1009-12.

2. Turk MJ, Guevara-Patiño JA, Rizzuto GA, Engelhorn ME, Sakaguchi S, Houghton AN. Concomitant tumor immunity to a poorly immunogenic melanoma is prevented by regulatory T cells. J Exp Med 2004;200(6):771-82. 